\title{
Another Extension of Heinz's Inequality Marvin Marcus
}

(December 6, 1960)

\begin{abstract}
A recent result of Heinz gives bounds on the bilinear form associated with a matrix $Q$ in terms of bounds on the two Hermitian parts of $Q$. This is extended to certain determinants associated with $Q$ by use of the Grassmann algebra.
\end{abstract}

Let $A$ and $B$ be non-negative Hermitian (n.n.h.) $n$-square matrices and let $Q$ be an arbitrary $n$-square matrix. A recent inequality due to E. Heinz [3] ${ }^{1}$ states that if $A^{2}-Q^{*} Q \geq 0$ and $B^{2}-Q Q^{*} \geq 0$, then

$$
|(Q u, v)| \leq\left\|A^{p} u\right\|\left\|B^{1-p} v\right\|, \quad 0 \leq p \leq 1
$$

sor any $u$ and $v$. Here $X \geq 0$ means simply that $X$ s non-negative Hermitian. The most recent proof of (1) is found in [1] along with several references to other proofs and extensions.

In this paper the following generalization of (1) will be presented.

Theorem. If $A^{2}-Q^{*} Q \geq 0$ and $B^{2}-Q Q^{*} \geq 0$ and $u_{1}, \ldots, u_{k}, v_{1}, \ldots, v_{k}$ are any $2 k$ vectors, $1 \leq k \leq n$, then

$$
\begin{aligned}
&\left|\operatorname{det}\left\{\left(Q u_{i}, v_{j}\right)\right\}\right|^{2} \leq \operatorname{det}\left\{\left(A^{p} u_{i}, A^{p} u_{j}\right)\right\} \\
& \times \operatorname{det}\left\{\left(B^{1-p} v_{i}, B^{1-p} v_{\jmath}\right)\right\} \\
& \leq \prod_{1}^{k}\left\|A^{p} u_{i}\right\|^{2}\left\|B^{1-p} v_{i}\right\|^{2},
\end{aligned}
$$

where $0 \leq p \leq 1$.

In what follows we use certain elementary properties of the $k$ th compound matrix of $A, C_{k}(A)$, which are found in [5].

Lemma. If $H \geq 0$ and $K \geq 0$ and $H-K \geq 0$, then $C_{k}(H)-C_{k}(K) \geq 0$.

Proof. We may assume $K$ is nonsingular (and hence $H$ will be) by the standard continuity argument. Now $H-K \geq 0$ if and only if $H^{-1 / 2}(H-K) H^{-1 / 2}$ $=I-H^{-1 / 2} K H^{-1 / 2} \geq 0$. This latter inequality holds if and only if every eigenvalue of $H^{-1 / 2} K H^{-1 / 2}$ is at most 1 . Now the eigenvalues of $C_{k}\left(H^{-1} K\right)$ are the $\left(\begin{array}{l}n \\ k\end{array}\right)$ products taken $k$ at a time of the eigenvalues of $H^{-1} K$. Moreover $H^{-1} K$ has non-negative eigenvalues and hence every eigenvalue of $C_{k}\left(H^{-1} K\right)$ is bounded by 1 . Now $\mathrm{C}_{k}\left(H^{-1} K\right)=C_{k}\left(H^{-1}\right) C_{k}(K)$ and this latter matrix is similar to $\left[C_{k}(H)\right]^{-1 / 2} C_{k}(K)\left[C_{k}(H)\right]^{-1 / 2}$. Thus

$$
C_{k}(I)-\left[C_{k}(H)\right]^{-1 / 2} C_{k}(K)\left[C_{k}(H)\right]^{-1 / 2} \geq 0
$$

and

$$
C_{k}(H)-C_{k}(K) \geq 0
$$

For completeness we next include a very brief and elementary proof of (1) which relies on the fact [4] that $\varphi(\lambda)=\lambda^{p}, \quad 0 \leq p \leq 1$, is monotone of every order

\footnotetext{
1 Figures in brackets indicate the literature references at the end of this paper.
}

for non-negative $\lambda$. A scalar function $\varphi$ is said to be monotone of order $n$ on $a \leq \lambda \leq b$ if whenever $H$ $-K \geq 0$ it follows that $\varphi(H)-\varphi(K) \geq 0$, where $H$ and $K$ are $n$-square Hermitian with eigenvalues in the interval $a \leq \lambda \leq b$. To see (1) let $Q=U H$ be the polar factorization of $Q, H \geq 0, \quad U$ unitary. Then the hypotheses are equivalent to $A^{2}-H^{2} \geq 0$, $B^{2}-\left(U H U^{*}\right)^{2} \geq 0$. Setting $w=U^{*} v$ we compute that

$$
\begin{aligned}
|(Q u, v)|^{2} & =|(H u, w)|^{2}=\left|\left(H^{p} H^{1-p} u, w\right)\right|^{2} \\
& =\left|\left(H^{p} u, H^{1-p} w\right)\right|^{2} \leq\left(H^{2 p} u, u\right)\left(H^{2(1-p)} w, w\right) \\
& =\left(H^{p} u, H^{p} u\right)\left(U H^{1-p} U^{*} v, U H^{1-p} U^{*} v\right) \\
& \leq\left(A^{p} u, A^{p} u\right)\left(B^{1-p} v, B^{1-p} v\right) \\
& =\left\|A^{p} u\right\|^{2}\left\|B^{1-p} v\right\|^{2} .
\end{aligned}
$$

To proceed to the proof of (2) let $u_{1} \wedge \ldots \wedge u_{k}$ denote the Grassmann (outer) product [2] of the vectors $u_{1}, \ldots ., u_{k}$. Then, by the lemma,

$$
0 \leq C_{k}\left(A^{2}\right)-C_{k}\left(Q^{*} Q\right)=C_{k}^{2}(A)-C_{k}^{*}(Q) C_{k}(Q)
$$

and

$$
0 \leq C_{k}^{2}(B)-C_{k}(Q) C_{k}^{*}(Q) .
$$

Hence, applying (1) to $C_{k}(Q), \quad C_{k}(A)$, and $C_{k}(B)$ in place of $Q, A$, and $B$ respectively, we have

$$
\begin{aligned}
\left|\operatorname{det}\left\{\left(Q u_{i}, v_{j}\right)\right\}\right|^{2} & =\left|\left(C_{k}(Q) u_{1} \wedge \ldots \wedge u_{k}, v_{1} \wedge \ldots \wedge v_{k}\right)\right|^{2} \\
& \leq\left\|\left[C_{k}(A)\right]^{p} u_{1} \wedge \ldots \wedge u_{k}\right\|^{2} \\
& \left\|\left[C_{k}(B)\right]^{1-p} v_{1} \wedge \ldots \wedge v_{k}\right\|^{2} \\
& =\left\|C_{k}\left(A^{p}\right) u_{1} \wedge \ldots \wedge u_{k}\right\|^{2} \\
& =\left\|A^{p} u_{1} \wedge \ldots \wedge A^{p} u_{k}\right\|^{2} \\
& =\operatorname{det}\left\{\left(A^{p} u_{i}, A^{p} u_{j}\right)\right\} \\
& \times \operatorname{det}\left\{\left(B^{1-p} v_{i}, B^{1-p} v_{j}\right)\right\} \\
& \leq \prod_{i=1}^{k}\left\|A^{p} u_{i}\right\|^{2}\left\|B^{1-p} v_{i}\right\|^{2} .
\end{aligned}
$$


This last inequality is an application of the Hadamard determinant inequality to $\left\{\left(A^{p} u_{i}, A^{p} u_{j}\right)\right\}$ and $\left\{\left(B^{1-p} v_{i}, B^{1-p} v_{j}\right)\right\}$ and completes the proof.

If $1 \leq i_{1}<\ldots<i_{k} \leq n$ and $1 \leq j_{1}<\ldots<j_{k} \leq n$ are sequences of integers, then $A\left[\bar{i}_{1}, \ldots, i_{k} \mid j_{1}, \ldots, j_{k}\right]$ will denote the $k$-square submatrix of $A,\left(a_{i_{s} j_{t}}\right)$, $s, t=1, \ldots, k$.

Corollary 1. If $A^{2}-Q^{*} Q \geq 0$ and $B^{2}-Q Q^{*} \geq 0$ and $0 \leq p \leq 1$, then

$\left|\operatorname{det} Q\left[j_{1}, \ldots, j_{k} \mid i_{1}, \ldots, i_{k}\right]\right|^{2} \leq$

$$
\begin{aligned}
& \operatorname{det} A^{2 p}\left[i_{1}, \ldots, i_{k} \mid i_{1}, \ldots, i_{k}\right] \\
& \operatorname{det} B^{2(1-p)}\left[j_{1}, \ldots, j_{k} \mid j_{1}, \ldots, j_{k}\right] .
\end{aligned}
$$

Proof. Let $u_{s}=e_{i_{s}}, \quad v_{s}=e_{j_{s}}, \quad s=1, \ldots ., k$ where $e_{t}$ is the unit vector with 1 in position $t, 0$ elsewhere.

Let $A$ and $B$ have eigenvalues $\alpha_{1} \geq \ldots \geq \alpha_{n}$ and $\beta_{1} \geq \ldots . \beta_{n}$ respectively. A bound for the left hand member of (2) may be given in terms of these eigenvalues as follows.

Corollary 2. Under the conditions of the theorem $\left|\operatorname{det}\left\{\left(Q u_{i}, v_{j}\right)\right\}\right| \leq \prod_{1}^{k} \alpha_{i}^{p} \beta_{i}^{1-p}\left(\operatorname{det}\left\{\left(u_{i}, u_{j}\right)\right\}\right)^{1 / 2}$

$$
\left(\operatorname{det}\left\{\left(v_{i}, v_{j}\right)\right\}\right)^{1 / 2} \text {. }
$$

Proof. Note that

$$
\begin{aligned}
\operatorname{det}\left\{\left(A^{2 p} u_{i}, u_{j}\right)\right\} & =\left\|C_{k}\left(A^{p}\right) u_{1 \wedge} \cdots \wedge u_{k}\right\|^{2} \\
& \leq \prod_{i=1}^{k} \alpha_{i}^{2 p}\left\|u_{1 \wedge} \cdots \wedge u_{k}\right\|^{2} \\
& =\prod_{i=1}^{k} \alpha_{i}^{2 p} \operatorname{det}\left\{\left(u_{i}, u_{j}\right)\right\} .
\end{aligned}
$$

Applying this to (2) we get (3).

\section{References}

[1] H. O. Cordes, A matrix inequality, Proc. Am. Math. Soc. 11, 206-210 (1960).

[2] W. Gröbner, Matrizenrechnung, p. 28 (München, 1956).

[3] E. Heinz, Beiträge zur Störungstheorie der Spektralzerlegung, Math. Ann. 123, 415-438 (1951).

[4] C. Loewner, Some classes of functions defined by difference or differential inequalities, Bull. Am. Math. Soc. 56, 308-319 (1950).

[5] J. H. M. Wedderburn, Lectures on matrices, Am. Math. Soc. Colloq. Publ. 17, 64 (1934).

(Paper 6582-51) 